\title{
Investigation into Solute Stabilizing Effects in Nanocrystalline Materials: An Atom Probe Characterization Study
}

Gregory B. Thompson ${ }^{1}$, Monica Kapoor ${ }^{1}$, Tyler Kaub ${ }^{1}$, Brad Boyce ${ }^{2}$, Blythe Clarke ${ }^{2}$, Kris Darling $^{3}$, Peter Felfer ${ }^{4}$, and Julie Cairney ${ }^{4}$

${ }^{1}$ The University of Alabama, Tuscaloosa, AL 35487 USA

${ }^{2}$ Sandia National Laboratories, Albuquerque, NM 87123 USA

${ }^{3}$ Army Research Laboratory, Adelphi, MD 20783 USA

${ }^{4}$ The University of Sydney, Sydney, NSW 2006 AUS

Over the past few years, there has been a concerted interest in understanding how solute segregation to grain boundaries stabilizes nanocrystalline materials against grain growth and stress effects [1-3]. To elucidate this behavior, the ability to quantitatively probe the chemistry of the grain boundary is essential. Atom probe tomography is ideally suited to achieve this level of atomic scale chemical analysis. This talk will address how atom probe is providing insights into solute segregation that leads to a variety of different nanocrystalline stabilization conditions.

The first case study involves the influence of adatom mobility during the growth of a thin film. Thin film stress evolves from an initial compressive state created as adatoms nucleate and form embryonic islands to a tensile state as these islands coalescence. For post-coalescence films, the stress can retain the tensile state for low mobility adataoms or become compressive for higher mobility adatoms. To date, the governing mechanisms for post-coalescence compressive stress evolution has not been well understood. Chason et al. [4] have proposed that excess adatoms in the grain boundaries create this post-coalescence compressive stress condition; to date, there has been little direct experimental evidence characterizing these excess atoms in the grain boundaries. A series of segregating alloy films, with different enthalpies of segregation, have been sputtered deposited. For a weakly segregating system, such as $\mathrm{Cu}(\mathrm{Ni})$, we have found that a small amount of solute is sufficient to dramatically increase the compressive stress, Figure 1, but whose effects are limited once grain boundary compositional saturation has occurred. Using interfacial excess atom probe reconstructions refined by Felfer et al. [5], we have quantified solute segregation at these boundaries to be $\Gamma_{\max } \sim 0.9$ atoms $/ \mathrm{nm}^{2}$ which will be elaborated on in how this intrinsic segregation regulates the overall film stress states.

In a synergistic study, we are investigating how solutes in similar boundaries can stabilize the grains themselves from growth with increasing temperature. $\mathrm{Cu}-\mathrm{Nb}$ is an anti-segregating system which has been used to address these thermal stability effects as an extreme case experimental example. A series of 'bulk' ball milled $\mathrm{Cu}(\mathrm{Nb}) \mathrm{x}$ alloys, where $\mathrm{X}$ varied from zero to ten at.\%, have been processed and annealed. The nanocrystalline grains were shown to be stabilized up to $400^{\circ} \mathrm{C}$, where upon evident clustering for the low solute concentrations was noted, Figure 2. Increasing the temperature resulted in phase separation for the higher alloy concentrations. These atom probe results are discussed in terms of the present thermodynamic [1] and mechanical stability [6] concepts of solute segregated stabilizing effects. 


\section{References:}

[1] H. A. Murdoch, C. A. Schuh. Stability of binary nanocrystalline alloys against grain growth and phase separation. Acta Materialia 61 (2013) 2121-2132.

[2] F. Tang, D.S. Gianola, M.P. Moody, K.J. Hemker, J.M. Cairney, “Observations of grain boundary impurities in nanocrystalline aluminum and their influence on microstructural stability and mechanical behavior," Acta Materialia 60 (2012), 1038

[3] B. Fu, G. B. Thompson. Compositional dependent thin film stress states. Journal of Applied Physics 108 (2010) 043506-1-6.

[4] E. Chason, B. Sheldon, et al. Origin of compressive residual stress in polycrystalline thin films. Physical Review Letters 88 (2002) 156103-1-4.

[5] P. Felfer, A. Ceguerra, S. Ringer, J. Cairney. Applying computational geometry techniques for advanced feature analysis in atom probe data. Ultramicroscopy 132 (2013) 100-106

[6] Darling K. A. Darling, B. K. VanLeeuwen, et al. Stabilized nanocrystalline iron-based alloys: Guiding efforts in alloy selection. Materials Science and Engineering: A 528 (2011) 4365-4371.

[7] GBT and TK acknowledge the AR0-W911NF1310436 grant; MK, BB and BC recognize The U.S. Department of Energy, Office of Science, Basic Energy Sciences, Materials Sciences and Engineering Division for support. Sandia National Laboratories is a multi-program laboratory managed and operated by Sandia Corporation, a wholly owned subsidiary of Lockheed Martin Corporation, for the U.S.

Department of Energy's National Nuclear Security Administration under contract DE-AC04-94AL85000

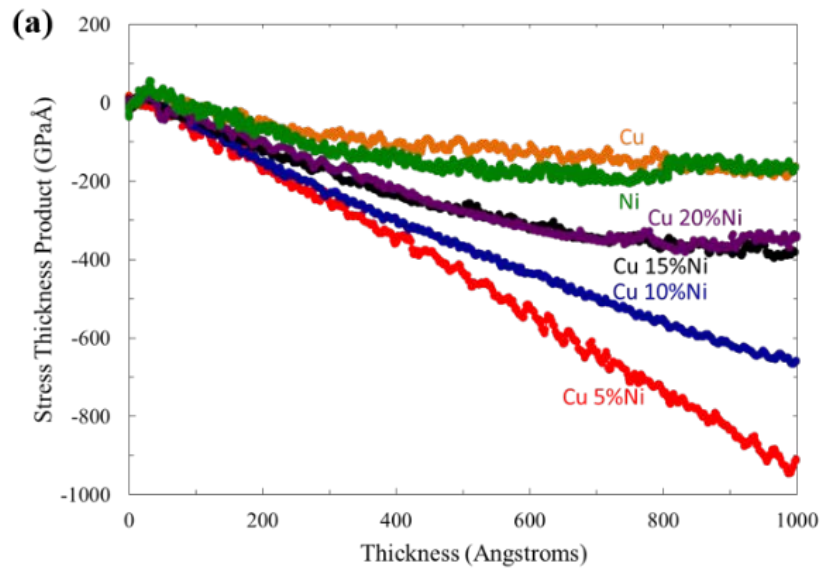

(b)

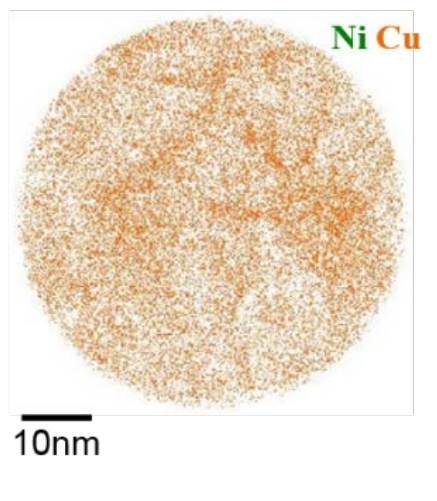

Figure 1: (a) In situ growth stress plot of $\mathrm{Cu}(\mathrm{Ni})$ films at various $\mathrm{Ni}$ solute content levels. Note that at 5 at.\% Ni the compressive stress was the largest and is contributed to the segregation of $\mathrm{Ni}$ to the grain boundaries. As the Ni content increased, atom probe revealed that the grain boundaries were solute saturated and the $\mathrm{Ni}$ was incorporated into the $\mathrm{Cu}$ matrix grains. (b) Atom map of $\mathrm{Cu}-5 \mathrm{at} . \% \mathrm{Ni}$

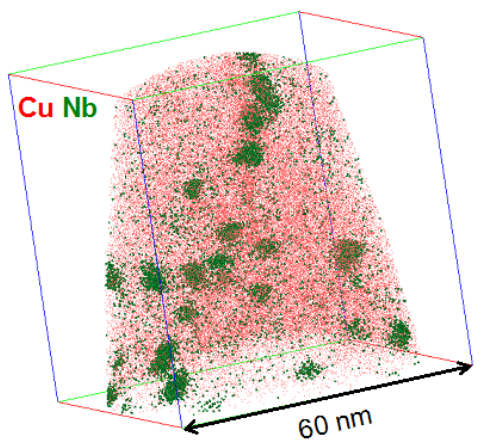

Figure 2: Atom map of $\mathrm{Cu}-1 \mathrm{at} . \% \mathrm{Nb}$ annealed at $400^{\circ} \mathrm{C}$ revealing $\mathrm{Nb}$ clustering at the grain boundaries. 